\title{
La spécificité de la Zone dite « rattachée à Bruxelles »
}

\section{Monique Heddebaut}

\section{(2) OpenEdition}

1 Journals

Édition électronique

URL : https://journals.openedition.org/tsafon/2743

DOI : $10.4000 /$ tsafon. 2743

ISSN : 2609-6420

Éditeur

Association Jean-Marie Delmaire

\section{Édition imprimée}

Date de publication : 1 juillet 2020

Pagination : 17-38

ISSN : 1149-6630

\section{Référence électronique}

Monique Heddebaut, « La spécificité de la Zone dite « rattachée à Bruxelles » », Tsafon [En ligne], 79|

2020, mis en ligne le 01 juillet 2020, consulté le 23 juin 2021. URL : http://journals.openedition.org/ tsafon/2743; DOI : https://doi.org/10.4000/tsafon.2743

Tsafon. Revues d'études juives du Nord 


\section{La spécificité de la Zone dite « rattachée à Bruxelles »}

\section{Monique Heddebaut*}

Après la torpeur de la «drôle de guerre », la «Sitzkrieg » ou « guerre assise » côté allemand, la Wehrmacht envahit le 10 mai 1940, sans le moindre ultimatum, les Pays-Bas, la Belgique, le Luxembourg, puis le Nord de la France dans une avancée qui surprend totalement le commandement allié, préparé à la seule guerre défensive. Au terme de ce qui fut qualifié avec un certain dédain de "Campagne des dix-huit jours » en Belgique avec capitulation le 28 mai 1940 et après la «bataille de France » qui prit fin le 18 juin, les territoires septentrionaux de la France sont immédiatement devenus zone de guerre et de front, car zone stratégique de la plus haute importance face à l'Angleterre.

\section{Le Nord - Pas-de-Calais, une situation particulière dès le printemps 1940}

\section{Le Militärbefehlshaber in Belgien und Nord-Frankreich (MBB)}

Les départements du Nord et du Pas-de-Calais sont immédiatement détachés de la France et intégrés dans une zone dirigée par le Militärbefehlshaber in Belgien und Nord-Frankreich (MBB) - le commandant militaire en Belgique et dans le Nord de la France - avant même la signature de l'armistice le 22 juin 1940. Le général Alexander von Falkenhausen, promu au rang de commandant, reçoit par la volonté expresse de Hitler, la responsabilité de gouverner les Pays-Bas dès le 16 mai 1940, la Belgique le 20 mai, puis le Luxembourg. Le commandant militaire se voit adjoindre les territoires envahis du Nord-Pas-de-Calais

\footnotetext{
* Musée de la Résistance de Bondues, Nord.
} 
considérés comme zone d'opérations militaires. À compter du 4 juin, le Nord et le Pas-de-Calais sont entièrement occupés. 120000 hommes, soit huit divisions, sont présents au lendemain de l'armistice. Ils exercent une répression particulièrement brutale.

La France est alors divisée en plusieurs zones suite à la convention d'armistice.

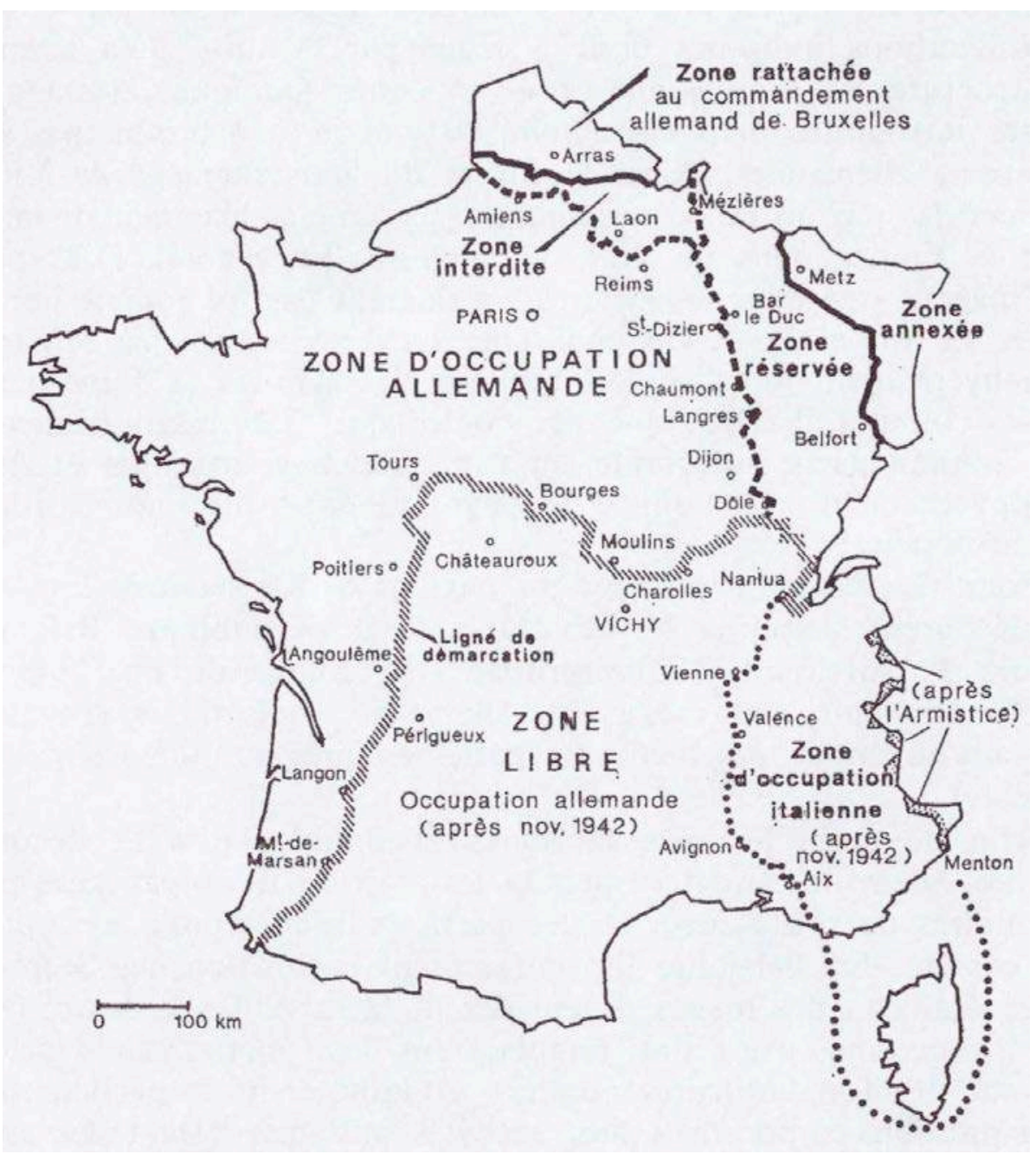

Les différentes zones qui divisent la France Revue du Nord, $\mathrm{n}^{\circ} 2$ hors série, 1985, p. 28.

Même si la Nordostlinie - la ligne du nord-est - qui délimite la Zone interdite et s'étend de la Somme à la frontière suisse, disparaît en 1941, le Nord de la France demeure tributaire des décisions de l'Oberfeldkommandantur (OFK 670) installée à Lille qui mène une politique autarcique agressive 


\section{GLIEDERUNG d. VERW. d. MIL.BEF. IN BELGIEN UND NORD-FRANKREICH}

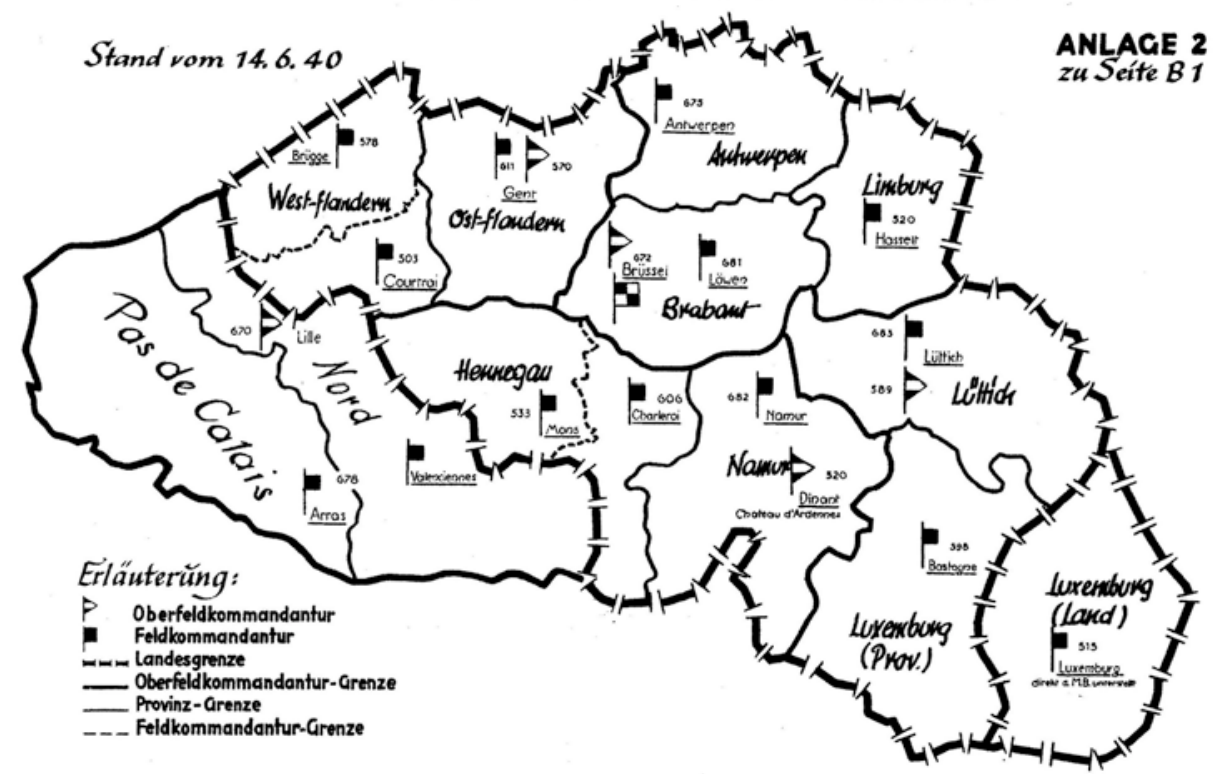

Divisions administratives du Militärbefehlshaber in Belgien und Nordfrankreich, 6 juin 1940

Bundesarchiv-MA, RW 36-202

La population va connaître de ce fait et au quotidien, dès le début de l'occupation militaire et jusqu'à la fin du conflit, mise au pas, répression, déportation, pillage et spoliation. Les habitants se vivent dès lors comme abandonnés et livrés aux vainqueurs allemands, dans un sentiment d'isolement profondément intériorisé dans les premiers mois qui ont suivi l'invasion et surtout dans la peur d'une annexion de facto de l'Alsace-Moselle ${ }^{1}$.

\section{Culture de guerre et anglophilie}

Les réticences sont sérieuses vis-à-vis du gouvernement réfugié à Vichy, même si le maréchal Pétain a fait «don de sa personne à la France ». Est immédiatement réactivée une culture de guerre, spécifique à la région, héritée de la Première Guerre, quand le Nord était occupé militairement à $70 \%$ et le Pas-de-Calais à 25\%. L'expérience et les souffrances passées ont imprégné la mémoire collective dans cette région

\footnotetext{
${ }^{1}$ Pour une histoire générale de la Deuxième Guerre mondiale dans la «Zone rattachée » à Bruxelles, lire Étienne Dejonghe, Yves Le Maner, Le Nord-Pas-de-Calais dans la main allemande. 1940-1944, Lille, Éditions La Voix du Nord, 2019, $2^{\mathrm{e}}$ édition, 431 p. Nous ne les citerons plus.
} 
tant chez ceux qui ont combattu, que chez ceux qui ont vécu en pays occupé durant tout le conflit, deux décennies plus tôt. La population avait déjà connu humiliations, pillage et exploitation économique, déportations, mais également un engagement dans des actes de résistance. Le personnage le plus emblématique fut Louise de Bettignies qui espionnait pour le compte de l'armée britannique et qui est décédée en septembre 1918 dans une prison à Cologne.

La phobie des francs-tireurs héritée de ce premier conflit était répandue dans toute l'armée allemande au point de soupçonner chaque civil d'être un combattant irrégulier en puissance. L'occupant est en effet sans état d'âme dans la Zone dite rattachée à Bruxelles. Lors de la première phase de l'invasion, 600 civils et prisonniers au moins sont abattus à la fin du mois de mai 1940, dans un territoire entre ArrasBéthune-Lille et Douai.

La région a pu développer une anglophilie certaine liée à la libération de ce territoire à l'automne 1918 par les Alliés, ce dont les Allemands sont parfaitement conscients. Cet engouement est largement réactivé lorsque la British Expeditionary Force (BEF) débarque le 12 septembre 1939 à Boulogne, après l'invasion allemande de la Pologne en 1939, alors que l'essentiel des troupes françaises est stationné en Lorraine et en Champagne.

Dès la mobilisation générale le gouvernement français reprend les principes adoptés en 1914 en ayant recours aux engagés volontaires étrangers au sein de la Légion étrangère, les Régiments de Marche de Volontaires Étrangers (RMVE). Nombreux ont à cœur de défendre leur patrie d'adoption contre la menace nazie, espérant également obtenir la nationalité française ou l'amélioration matérielle de la vie familiale. Ainsi, le $22^{\mathrm{e}}$ RMVE qui débarque le 5 juin 1940 à Villers-Cotterêts et combat autour de Soissons, ne compte pas moins de $30 \%$ de Juifs polonais et roumains. L'engagement des juifs de Lens dans ces régiments a été massif ${ }^{2}$. D'autres ont choisi d'intégrer un régiment «classique » de la Légion étrangère lorsque certains rejoignent l'armée polonaise.

Alors que la région est entièrement occupée, les premiers actes d'une résistance spontanée consistent en l'hébergement des soldats anglais arrivés en 1939. Puis, au-delà de la compassion et de la sympathie vis-à-vis des prisonniers emmenés par colonnes ou par convois en Allemagne, il s'agit de sauver et faire évader les rescapés des

\footnotetext{
2 Témoignage de Franck Fajnkuchen qui cite Georges Biezunski, Pejsach Sulman, Manek Fajnkuchen, 2019. Tapuscrit non édité.
} 
combats de mai-juin 1940, puis de récupérer les armes abandonnées. Des aviateurs anglais enfermés dans la «poche de Dunkerque » qui n'ont pu être réembarqués entre le 26 mai et le 4 juin 1940 lors de l'opération d'évacuation - du nom de code Dynamo - sont cachés par des habitants, ce qui vaut déjà dénonciations et incarcérations.

\section{Les marches germaniques de l'arc septentrional}

La France est devenue de facto un État satellite du Reich qui se met rapidement au diapason de la politique discriminatoire et raciale nationalsocialiste. Mais le découpage en zones, avec chacune sa spécificité, explique les différences de traitement et de calendriers pour les deux populations visées par le meurtre et l'esclavage-extinction : les juifs et les Tsiganes. L'ordre décisif vient de Hitler en décembre 1941. La conférence du 20 janvier 1942 à Wannsee met au point l'organisation administrative, technique et économique de l'Endlösung, la «Solution finale », sous l'autorité de Heydrich et sous le contrôle de la SS, pour débarrasser l'Europe de ses juifs, en un an. La France apparaît comme le principal territoire à déjudaïser avec 700000 juifs estimés.

Le régime d'exception qui s'est amplifié, s'explique par la situation géographique et stratégique de ces territoires face à l'Angleterre, mais aussi par leur poids économique avec un pillage et une surexploitation immédiate des ressources agricoles et industrielles, avec rafles de main d'œuvre. En 1939, 60\% de la production de houille et la moitié de la consommation électrique sont assurés par la région Nord. La répression a démarré très rapidement envers les communistes, les francs-maçons et les juifs, appartenant à l'anti-France pour le régime de Vichy, ennemis idéologiques pour l'occupant. Le «judéobolchévique » est l'ennemi par excellence. Avec l'ouverture d'un second front à l'ouest sur la côte atlantique et la décision de construire l'Atlantikwall - mur de l'Atlantique - et de puissants secteurs défensifs, s'ouvre une douzaine de camps. Ils ont fonctionné pendant vingt-huit mois entre Calais et Abbeville, gérés et surveillés par l'Organisation Todt (OT), réclamant une énorme main d'œuvre, sans cesse renouvelée, et utilisant à la fois des volontaires, des requis français, des détenus de droit commun, mais également des juifs belges qui vont ensuite compléter les convois de déportation.

Mais au-delà des considérations militaires et économiques, l'enjeu d'un tel découpage en zones correspond à la vision nazie d'une Europe nouvelle dans laquelle la Flandre et l'Artois auraient constitué des 
marches germaniques retrouvées, car issues d'un espace conquis, volé par Louis XIV qui a repoussé les frontières du Nord lors de la guerre de Dévolution en 1667.

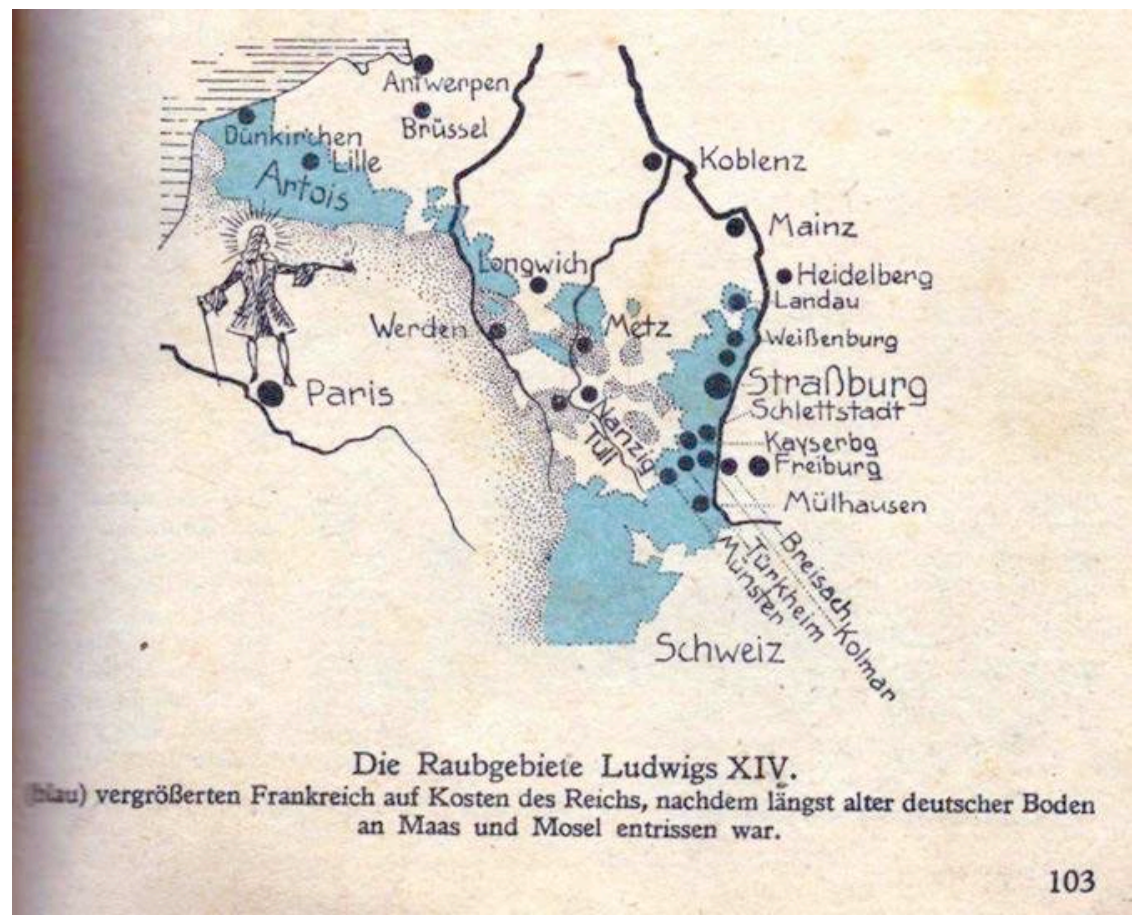

Les territoires volés par Louis XIV

Der Weg zum Reich, Livre de classe allemand,1944, p. 103

L'occupant qui a la volonté de créer une enclave ethnique germanophone, s'appuie sur l'association Volksdeutsche Sprachgemeinschaft Nordfrankreich - communauté de langue allemande du Nord de la France - créée en avril 1942. Les mineurs qui avaient déjà émigré une première fois de Pologne en Westphalie à la fin du XIX siècle avant de s'installer dans le bassin minier du Nord-Pas-de-Calais, les «Westphaliens », sont les cibles des idéologues nazis. Ces ouvriers sont considérés comme indicateurs potentiels de la SicherheitspolizeiSicherheitsdienst (Sipo-SD) - Police de sécurité-service de sécurité. Les autorités allemandes ont également cherché à utiliser les mouvements autonomistes minoritaires tels celui de l'abbé Jean-Marie Gantois, le Vlaamsch Verband van Frankrijk, et le docteur douaisien Quesnoy rallié aux théories raciales les plus extrêmes ${ }^{3}$.

\footnotetext{
${ }^{3}$ AD59, 1W1261, 1W1301, 1W758, 1W1294. Liste des abréviations en fin d'article.
} 


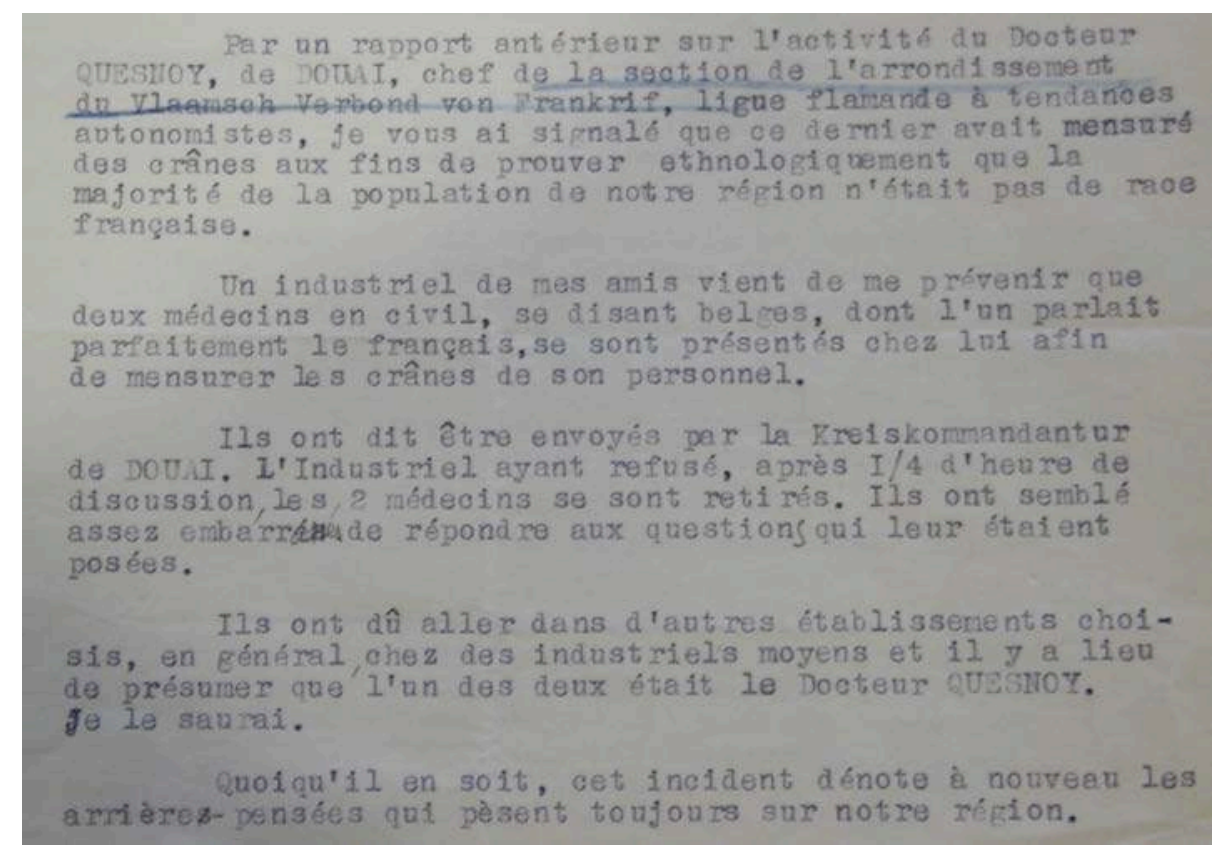

Rapport du sous-préfet de Douai reçu en préfecture le 4 septembre 1942

AD59, 1W1261

\section{Une déportation hors chronologie française et un sauvetage exceptionnel}

\section{La rafle du 11 septembre 1942}

Si les calendriers et les modalités d'exécution des mesures raciales et de déportation ont été discutés et mis au point dans les Zones occupée et libre entre les autorités allemandes et françaises, en revanche, en Zone rattachée, le calendrier est imposé par le $M B B$ en relation avec Berlin et en fonction de la date d'ouverture du centre de rassemblement des déportés raciaux, le Sammellager de Malines (Belgique), opérationnel dès le 27 juillet 1942.

La caserne Dossin installée entre Bruxelles et Anvers, l'antichambre d'Auschwitz, est l'équivalent de Drancy en France. Le premier convoi démarre le 4 août 1942. À l'instar de la rafle dite du Vel' d'Hiv' des 16 et 17 juillet 1942 à Paris qui a envoyé quelque 13000 juifs à Auschwitz via Drancy, une grande rafle est prévue et fixée au 11 septembre 1942, à la veille du Nouvel An juif, Roch Hashana. Des juifs 
étrangers, la plupart venus des pays de l'Est dans l'entre-deux-guerres pour contribuer à la reconstruction des territoires dévastés, sont arrêtés, regroupés et conduits à la gare de Lille-Fives, en attente d'un convoi à destination d'Auschwitz-Birkenau via le camp de Malines ${ }^{4}$.

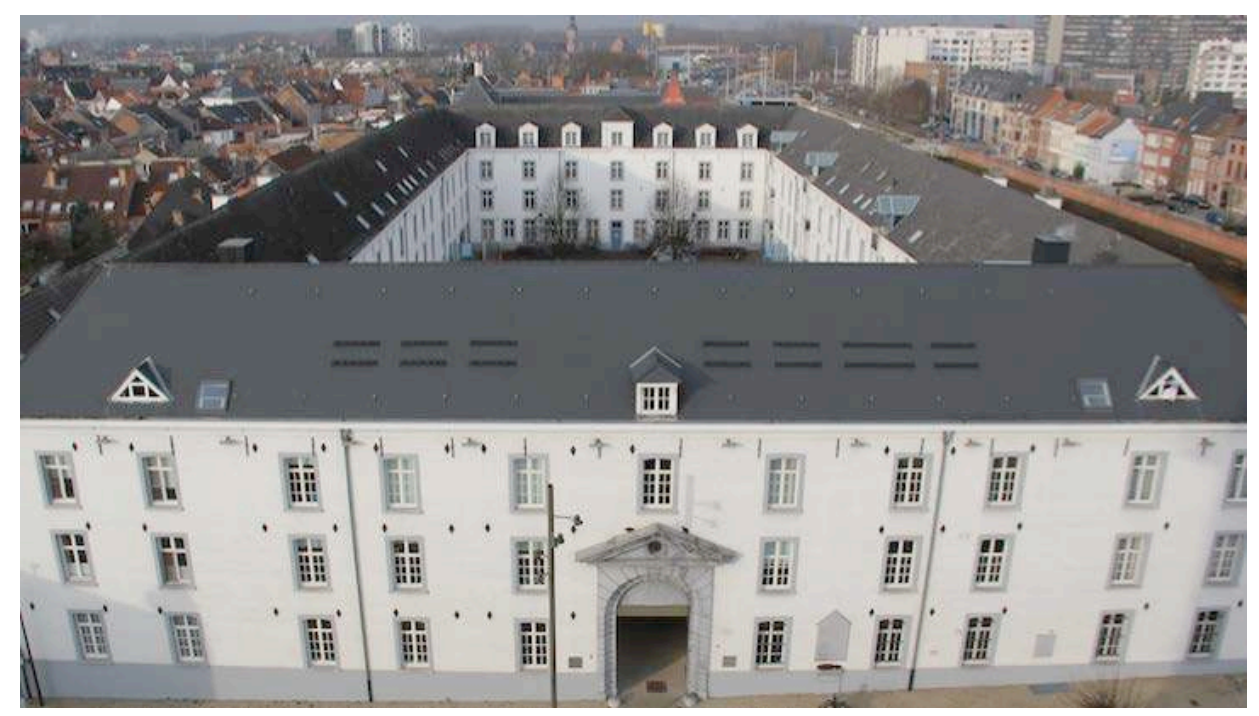

Malines, caserne Dossin, 2018

photo Monique Heddebaut

Mais sur 600 personnes environ dont la déportation a été programmée, les Allemands n'en recensent que 513. Selon les estimations actuelles quelque quatre-vingt personnes y ont échappé, soit qu'elles ont été prévenues de l'imminence du danger dans les jours qui ont précédé la rafle, la veille, dans la nuit et même au petit matin, ou qu'elles ont été sauvées essentiellement dans l'enceinte de la gare de Lille-Fives grâce à des cheminots aguerris et particulièrement bien organisés. Cela signifie au vu de ces chiffres qu'une autre résistance, moins spectaculaire, moins violente que la résistance armée et militaire, mais tout aussi efficace, a eu lieu pendant cet été 1942 particulièrement meurtrier pour les communautés juives.

\footnotetext{
${ }^{4}$ Voir la bibliographie en fin d'article.
} 


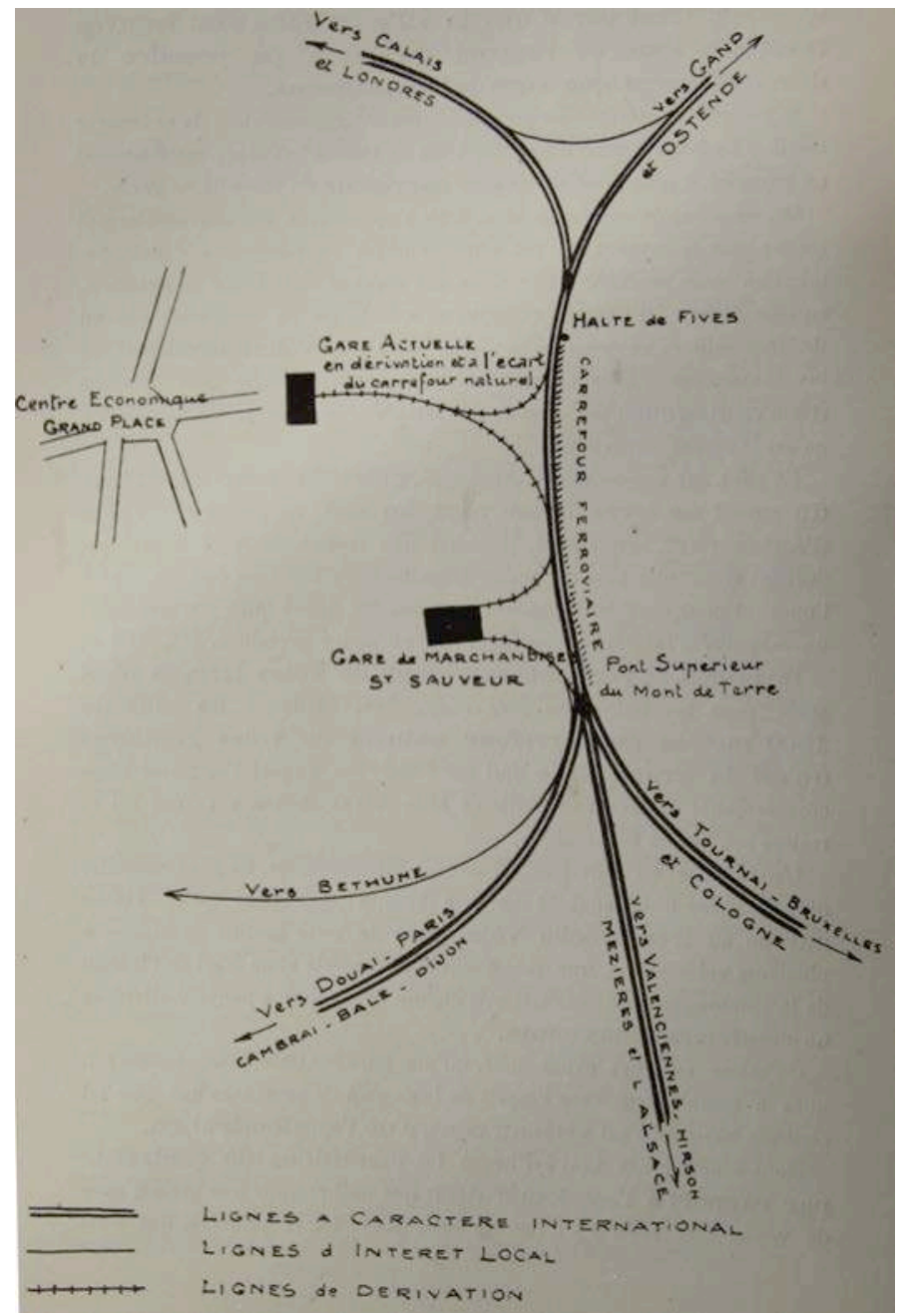

Gares de Lille en 1921

AML, 20-21

Et au lendemain du sauvetage qui eut lieu sur les quais de la gare de Lille-Fives s'est constitué le «Comité clandestin d'aide aux Juifs recherchés par la Gestapo de la région lilloise » car les enfants et les adultes, qui avaient échappé à la déportation, ne peuvent rester chez les cheminots dont certains domiciles sont perquisitionnés. Six personnes prennent la tête de ce Comité : d'une part les cheminots René Douce et Louis Saint-Maxent, et Paul Smekens - un résistant belge - tous trois membres du mouvement Voix du Nord et d'autre part Léon Leser, Simon Prechner et Alexandre Raby, juifs tous les trois. Le Comité a été opérationnel et effectif jusqu'à la fin de la guerre : une liste conservée par la famille Leser fait état de 167 personnes aidées et cachées entre 1942 et 
$1944^{5}$, alors que le mouvement Voix du Nord est décapité au tournant des années 1942-1943. Ses fondateurs sont arrêtés : Jules Noutour est torturé et envoyé au camp de Gross Rosen où il décède. Natalis Dumez est lui aussi déporté. Charles Deconninck est emprisonné à la forteresse de Gross-Strehlitz (Haute-Silésie), puis au camp de Sachsenhausen. René Douce qui avait été élu président du Comité de sauvetage immédiatement au lendemain de la rafle, est arrêté en décembre 1942 pour activité communiste. D'abord incarcéré à la centrale d'Eysses (Tarn-et-Garonne), il est déporté à Dachau en février 1944, pour avoir participé au soulèvement contre les miliciens et les Gardes mobiles et à une tentative d'évasion.

\section{La Résistance sans armes ...}

Vingt-quatre cheminots - voire 25 - couverts par leur chef de gare Jean Mabille ont participé aux opérations de sauvetage en gare de LilleFives le 11 septembre 1942. Pour la réussite de ce sauvetage au sein même de la gare d'embarquement pour Auschwitz via Malines, ces hommes sont épaulés dans et par des réseaux de résistance, par des hommes et des femmes issus de différents horizons politiques, professionnels, de confessions diverses, à la fois dans les milieux protestants et catholiques. Ils ont bénéficié de complicités en amont et en aval de la rafle, dans ce quartier de Fives, avec un efficace maillage de terrain, mais également de la part d'Allemands antifascistes ${ }^{6}$.

Certains de ces cheminots présents ce 11 septembre 1942 appartiennent au mouvement de résistance Voix $d u$ Nord qui a pris naissance en avril 1941 dans ce quartier de Lille-Fives et s'y sont engagés dès sa fondation : Louis Saint-Maxent, Marcel Deschryver et Marcel Thumerel. Leur implication est intiment liée à la vie de ce quartier industriel et ouvrier. Ils appartiennent au groupe de l'abbé Charles Deconninck, l'un des fondateurs du mouvement, nommé vicaire à l'église Notre-Dame de Lourdes à Hellemmes, dans l'immédiate proximité de la gare de Lille-Fives. Non seulement le groupe assure la reproduction et la diffusion de leur journal clandestin ${ }^{7}$ sur tout le réseau

\footnotetext{
${ }^{5}$ Sur cet épisode exceptionnel du sauvetage des juifs en gare de Fives et sur le Comité de clandestin, lire Monique Heddebaut, «Sans armes face à la rafle du 11 septembre 1942 », Tsafon, revue d'études juives du Nord, n 70, automne 2015-hiver 2016, p. 119168.

${ }^{6}$ Ibid.

${ }^{7}$ Ce journal clandestin du mouvement Voix du Nord est devenu le journal régional éponyme à la Libération.
} 
ferroviaire de la région Nord, mais encore, l'un d'eux, Pierre Hachin, fournit des listes du personnel S.N.C.F. truffées d'erreurs à la direction des transports de la Wehrmacht et surtout, renseigne la Résistance sur les horaires de tous les transports militaires allemands de passage dans la région, avec indication exacte de leur composition ${ }^{8}$. Les cheminots qui ne sont pas dupes de la soi-disant mise au travail des juifs, pressentent que le départ de ce convoi ne signifie qu'un aller simple pour les déportés.

En effet, très tôt, dès novembre 1941, et à plusieurs reprises, ce mouvement a pris clairement position contre les mesures antisémites du gouvernement de Vichy. Et après les arrestations, dans le numéro du 17 septembre 1942, ces résistants adressent un «hommage à la population française qui a manifesté son hostilité envers les Allemands et leurs [plats] serviteurs, à ceux qui ont facilité l'évasion et la mise à l'abri des Juifs ». Ils incitent leurs lecteurs et sympathisants à s'impliquer dans la prise en charge de tous ceux qui ont réussi à échapper à la déportation. Ils avancent le nombre de 50 [59 ?] enfants juifs sauvés à Lille et la présence de 103 agents de police - très proche des 105 cité par le commissaire de police de Lille - envoyés en renfort pour seconder les Allemands 9 .

Certains résistants de la Voix $d u$ Nord apportent leur concours à d'autres organisations, tel le mouvement de résistance « Rail », d'autres au mouvement Sylvestre-Farmer War Office (W.O.) qui agit par ailleurs pour le Bureau Central de Renseignements et d'Action (B.C.R.A.), à l'Organisation civile et militaire (O.C.M.). Ils ont parfois été adhérents d'un parti politique, à la Section française de l'Internationale ouvrière (S.F.I.O.) ou membres du parti communiste devenu clandestin. Le vingtcinquième cheminot du dépôt des machines de Lille-Fives, un communiste récemment identifié, a été chargé entre autres missions, de la traduction et de la diffusion de tracts en allemand à destination des troupes d'occupation.

\section{... au-delà du monde du rail}

Les policiers sont des auxiliaires et des relais précieux pour prévenir les responsables des communautés juives des rafles quand bien même les Allemands se méfient des Français en ne communiquant les ordres de réquisition et les dates des rafles aux commissariats qu'au

\footnotetext{
${ }^{8}$ André Diligent, Un cheminot sans importance, Paris, éditions France-Empire, 1975, $253 \mathrm{p}$.

${ }^{9}$ Rapport du commissaire de police de Lille daté du 11 septembre 1942 qui rend compte de la rafle, AD59, $1 \mathrm{~W}$ 1844. Présenté et analysé par Danielle Delmaire, Tsafon, revue d'études juives du Nord, n 72, automne 2016 - hiver 2017, p. 159-164.
} 
dernier moment. L'un d'eux a hébergé jusqu'à la fin de la guerre une fillette placée par ses parents qui redoutaient leur arrestation ${ }^{10}$.

Le mouvement Voix $d u$ Nord a tissé des liens avec l'Église protestante de Lille-Fives et les réseaux catholiques. L'un des maillons de cette chaîne de solidarité est un Allemand, le pasteur Friedrich Günther, un soldat-interprète en poste à Lille, à l'OFK 670. Ses positions religieuses l'ont amené à militer au sein de l'Église confessante (Bekenntniskirche) animée par Karl Barth. Il connaît bien à ce titre ses collègues de la métropole lilloise: les pasteurs Pasche à Roubaix et Cheradame à Lille qu'il prévient en cas de nécessité. Par son poste, à l'accueil et à la distribution courrier de la Kommandantur, à Lille, il détient des informations-clés sur les mesures en vigueur ou à venir et notamment sur les dates des rafles. Il l'a payé de sa vie puisque, jugé défaitiste et déserteur par l'un de ses supérieurs, il est grièvement blessé le 28 août 1944 dans les bureaux de l'OFK 670 sur le départ. Il est décédé deux jours plus tard à l'hôpital Calmette de Lille ${ }^{11}$. Deux missions populaires ou « postes d'évangélisation » qui existent à Roubaix et à Fives autour des pasteurs Henri Nick et Philippe Blanc ont pénétré le milieu ouvrier.

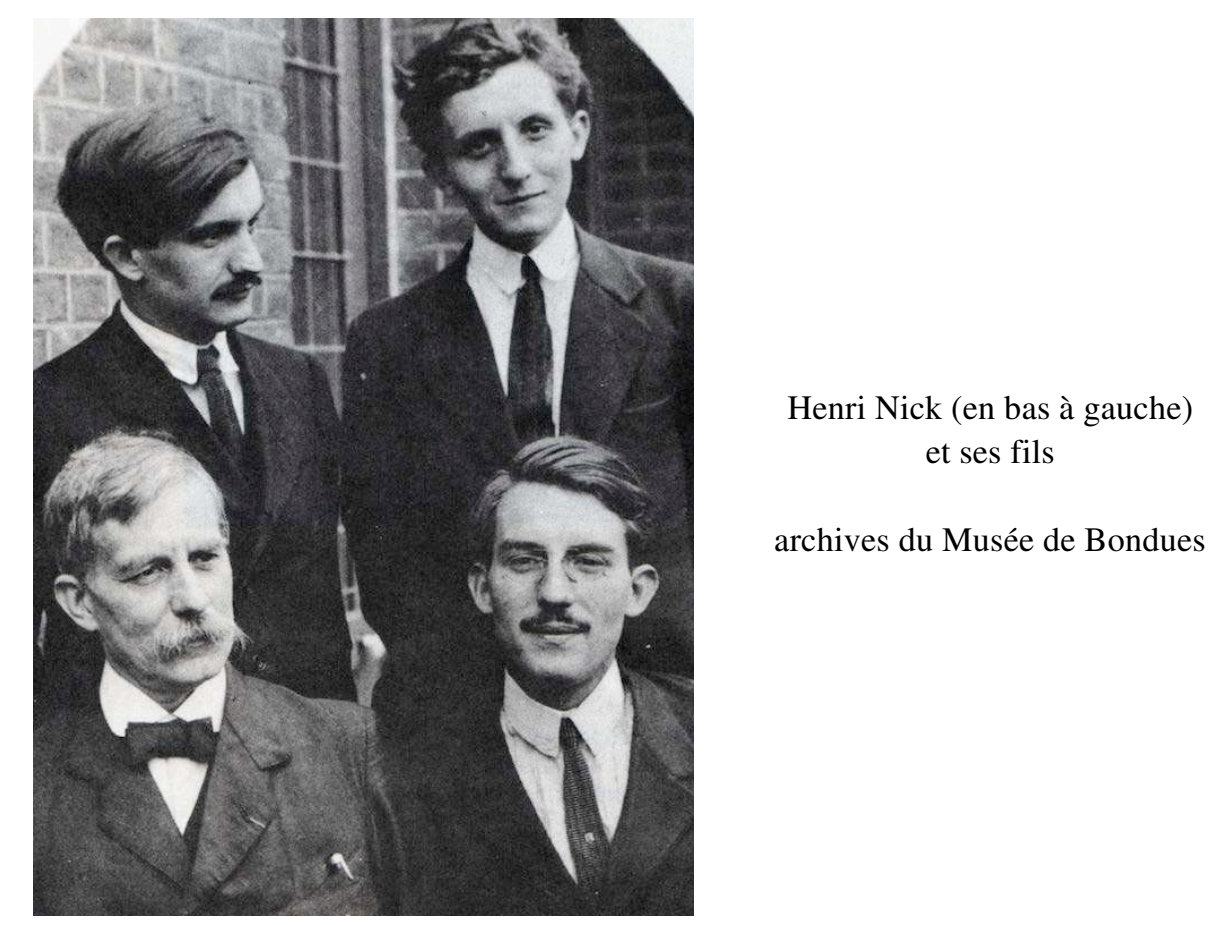

\footnotetext{
${ }^{10}$ Témoignage de Suzanne Meller née Karmiol, 2016.

${ }^{11}$ Témoignage de Marcel Pasche, Liens protestants, Hors-série juin 2014, p. 2-3.
} 
De formation semblable et avec les mêmes références, une autre institution joue un rôle important dans le sauvetage : la Maison de santé Ambroise Paré dirigée par Éva Durrleman et Thèrèse Matter, qui est très active dans le réseau W.O. Sylvestre Farmer. Les fils du pasteur Nick également membres de la Voix du Nord, accueillent des rescapés de la rafle à Inchy dans le Cambrésis, une vieille terre de protestantisme, là où l'Église réformée a un consistoire. Même si une fraction des fidèles est pétainiste, une autre partie s'est engagée dans la Résistance, d'abord dans les filières pour cacher et faire évader les aviateurs anglais et américains.

Si la hiérarchie catholique nordiste des diocèses de Lille, Arras et Cambrai a été d'un silence assourdissant ${ }^{12}$, il n'en a pas été de même pour certains prêtres. L'abbé Stahl, un avocat devenu prêtre, issu de la bourgeoisie industrielle lilloise, utilise son vaste réseau de connaissances pour cacher et sauver des réfractaires, des aviateurs anglais et surtout vingt et un juifs, adultes et enfants. Il réussit à les placer dans différentes institutions, au patronage du Buisson à Marcq-en-Barœul, à Bouvines, à Bapaume et surtout à les transférer vers d'autres adresses en cas de perquisitions. Ainsi le préventorium de Trélon dans l'Avesnois, dirigé par Jeanne Rousselle et secondée par Anne-Marie Capitain, ne cache pas moins de 54 personnes entre le $1^{\text {er }}$ août 1942 et le 3 juillet 1944.

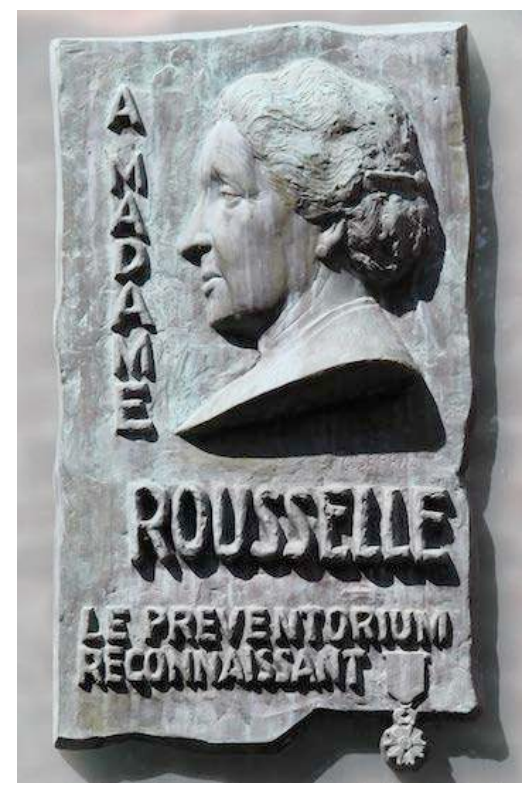

\author{
Maison des Enfants à Trélon \\ Plaque en hommage à \\ Jeanne Rousselle, \\ fondatrice de la Maison en 1923 \\ photo Monique Heddebaut
}

\footnotetext{
${ }^{12}$ Voir Danielle Delmaire, «Le cardinal Liénart devant la persécution des Juifs de Lille », dans André Kaspi, Serge Klarsfeld, Georges Wellers (sous la dir.) La France et la Question juive 1940-1944, Paris, S. Messinger, 1981, p. 231-248.
} 
Certains enfants ont été amenés juste avant la rafle du 11 septembre ou dans les semaines qui ont suivi, par l'intermédiaire de Mme Signoret, l'épouse de l'inspecteur d'académie, par les directrices de la clinique Ambroise Paré ou encore par maître Pacôme du mouvement Voix du Nord.

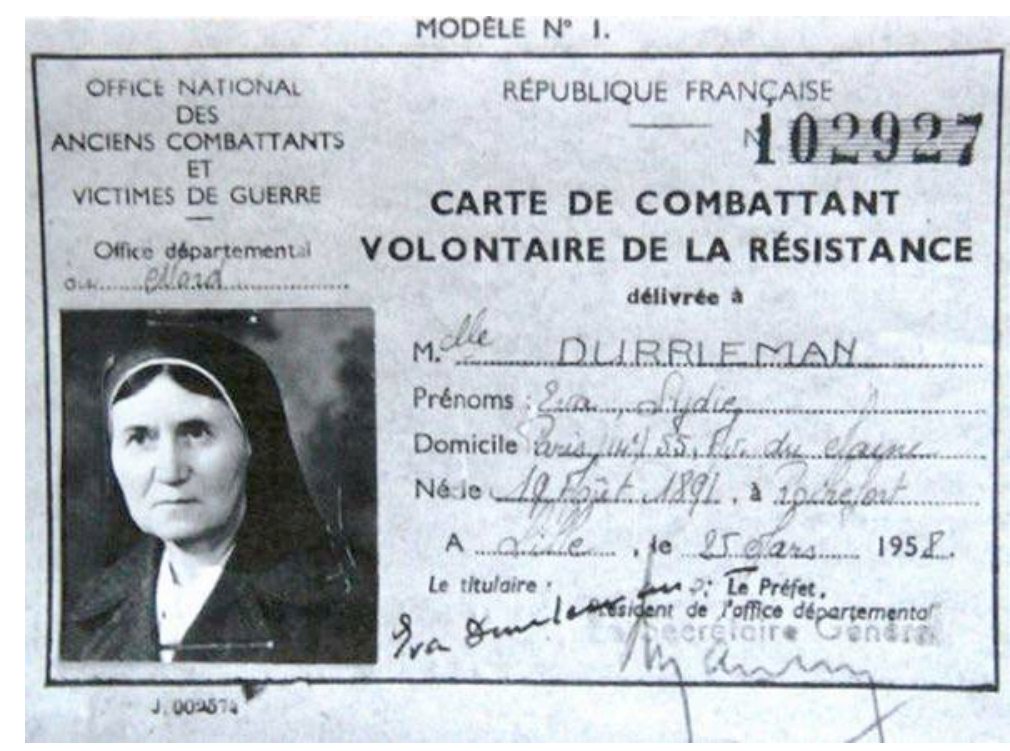

Carte de Combattant Volontaire de la Résistance d'Éva Durrleman

archives de la paroisse protestante de Lille-Fives

Jeanne Rousselle travaille de concert avec des résistants de la région lilloise et du Nord, mais également avec Denise Milhaud et Arlette Beaumont de l'Entraide Temporaire de Paris qui placent vingttrois enfants juifs de la région parisienne au préventorium entre mars 1943 et la Libération. Cette œuvre privée, clandestine et multiconfessionnelle, pour enfants s'est constituée en 1941 à l'ombre du Service social d'Aide aux Émigrants (SSAE). Fondée avec le concours de militantes catholiques, protestantes et juives, elle s'est prioritairement consacrée à partir de 1942 au sauvetage des enfants juifs privés de leurs parents, les faisant transiter vers des placements clandestins organisés dans des familles ou des institutions religieuses, souvent même à l'insu des responsables de l'Union Générale des Israélites de France (UGIF). Denise Milhaud assure la fonction d'assistante sociale en chef des centres d'enfants et des dispensaires gérés par l'UGIF aux côtés de son mari, 
Alfred Milhaud, qui était médecin à l'hôpital du Val-de-Grâce avant son éviction suite aux lois raciales ${ }^{13}$.

En soutien et en renfort de ces réseaux constitués opèrent certaines personnalités. Ainsi le commandant de la Kreiskommandantur d'Avesnes-sur-Helpe averti de l'arrestation d'une famille juive par un résistant du mouvement Voix $d u$ Nord, Charles Pacôme, intervient personnellement. Il réussit à joindre par radio devant la gare de Valenciennes le conducteur du camion qui a embarqué les familles raflées dans le sud du département du Nord. La famille Waisberg échappe ainsi à la déportation et se réfugie immédiatement en Zone non occupée grâce à ses faux papiers ${ }^{14}$.

Les relations de Thérèse Land, l'épouse du préfet Carles, née dans une famille juive non pratiquante, permettent à celle-ci d'obtenir secours et aides pour les personnes cachées. Ses tantes maternelles et sa grandmère ont été arrêtées lors de la rafle dite du Vel' d'Hiv', ce qui explique son implication. Jeanne Signoret-Vasserot, l'épouse de l'inspecteur d'académie parti en retraite car jugé trop tiède par le gouvernement de Vichy, membre de la S.F.I.O. lilloise, apporte son concours aux différentes étapes du sauvetage. Des entrevues avec le Comité de sauvetage se sont déroulées dans ses appartements ou ceux de Mme Carles. Y ont été discutées les questions matérielles et financières, non des moindres en ces périodes de pénurie et de rationnement ${ }^{15}$. Suzanne Legry-Nuez $^{16}$, autre membre influente de la S.F.I.O. et résistante de la première heure du mouvement Voix du Nord, s'occupe de la diffusion et même de l'impression du journal clandestin à la ronéo dans les locaux de la mairie de Lille. Lors de la rafle, cette dernière prévient les familles, s'occupe de leur hébergement et de leur ravitaillement ${ }^{17}$. Elle garde deux personnes recherchées pendant plusieurs mois chez elle. Le Comité de sauvetage est assisté par Félicien Hautcœur, chef du service du ravitaillement de la mairie de Lille, lui-même engagé dans le mouvement Libération-Nord, et par d'autres employés de la mairie ${ }^{18}$.

\footnotetext{
${ }^{13}$ Michel Laffitte, «L'UGIF, collaboration ou résistance ? », Revue d'Histoire de la Shoah, vol. 185 , no. 2, 2006, p. 45-64.

${ }^{14}$ Témoignages de Paulette et Jacqueline Waisberg, 2015.

15 AD59, 170W71074, dossier Legry.

${ }^{16}$ Suzanne Legry-Nuez, sœur de Rachel Lempereur, députée socialiste du Nord de 1945 à 1958.

${ }^{17}$ AD59, 170W71074, dossier Legry.

${ }^{18}$ AD59, 170W71073, dossier Hautcœur.
} 
Il n'entre pas dans le cadre de cette communication de dresser la liste de tous ceux qui sont intervenus à chacun des échelons de ce maillage de terrain dans la résistance civile: de l'Allemand ou du policier qui ont détourné le regard pour ne pas voir, de tous les cheminots au-delà des vingt-cinq identifiés jusqu'aux hommes et femmes animés par leur foi, l'esprit patriotique ou leur idéal républicain, de tous les petits gestes d'entraide jusqu'aux placements dans des familles ou des institutions. Mais une étude à paraître en développera tous ces aspects.

\section{La déportation des Tsiganes : un cas unique en France ${ }^{19}$}

Les nazis vont pouvoir s'appuyer dans le Nord de la France et en Belgique sur des législations discriminatoires antérieures à l'invasion de mai 1940, qui ont été mises au service de la politique raciale du Troisième Reich, car tous les États européens avaient adopté une législation spécifique de contrôle, utilisant comme repoussoir le migrant étranger, et tout particulièrement le «Juif» et le «Bohémien». La France avait créé une catégorie spécifique, celle des Nomades, afin d'identifier et de regrouper au moyen de carnets anthropométriques ceux qui étaient appelés indistinctement Bohémiens, Romanichels, Gitans, Nomades. C'est la loi du 16 juillet 1912 relative à «l'exercice des professions ambulantes et la réglementation de la circulation des nomades ». Leur mode de vie était assimilé au vagabondage, à la criminalité et l'asociabilité. Quelque 30000 personnes ont relevé de ce statut en 1924. En Belgique, les méthodes anthropométriques et dactyloscopiques venues de France ont été appliquées pour identifier les étrangers, parmi lesquels les Tsiganes de passage. Cette méthode d'abord réservée aux étrangers criminels ou réputés dangereux a progressivement été généralisée après la Première Guerre mondiale. Les Tsiganes possédaient en outre une feuille de route renouvelable tous les trois mois.

La déclaration de guerre en 1939 a certes durci la situation des Nomades avec le décret français du 6 avril 1940 qui leur interdit la circulation sur la totalité du territoire métropolitain. En Belgique la zone côtière est interdite aux itinérants, sans qu'ils soient expressément visés. Mais ils ne sont ni assignés à résidence, ni internés, à la différence du reste de la France, où des camps ont été décidés par les autorités allemandes ou créées par le gouvernement de Vichy. Au lendemain de la

\footnotetext{
${ }^{19}$ Sur cette déportation, lire Monique Heddebaut, Des Tsiganes vers Auschwitz..., op. cité en bibliographie en fin d'article.
} 
publication du Statut des juifs par le gouvernement de Vichy, l'état-major allemand exige le 4 octobre 1940, l'internement des familles tsiganes Zigeuner - de la Zone occupée dans des camps surveillés et gérés par les autorités françaises. Sont visés les Nomades tels qu'ils sont définis par la loi de 1912. Entre octobre 1940 et mai 1946, plus de 6500 personnes, en majorité française dont un grand nombre d'enfants, sont internées dans plus d'une trentaine de camps pour Nomades situés sur l'ensemble du territoire métropolitain. Ce dispositif hétérogène conjugue à la fois la maîtrise des circulations itinérantes, la ségrégation ethnique et la répression des catégories de l'asocialité.

Dans le $M B B$ les autorités françaises, belges et allemandes ne mettent pas en place de camps, pas plus qu'aux Pays-Bas, ce qui a laissé aux intéressés un sentiment de sécurité tout illusoire et ce qui a favorisé les regroupements. Mais entre temps, un enchaînement de décisions dont l'épicentre se trouvait en Allemagne, précipite le sort des Tsiganes qui se trouvent progressivement pris au piège des législations discriminatoires antérieures.

La déportation des communautés tsiganes d'Allemagne a été décidée par Hitler avec l'Auschwitz Erlass, le décret d'Auschwitz du 16 décembre 1942, dont le texte n'a pas été conservé, mais que l'on connaît au travers de son décret d'application du 29 janvier 1943. Il s'agit là du point culminant et de l'aboutissement d'une longue tradition de ségrégation. Dès février 1943 commence alors leur déportation à Auschwitz-Birkenau dans le Zigeunerlager, le camp des Tsiganes, qui est encore en construction, pour " eine Aktion von einigen Wochen in ein Konzentrationslager» [une action de quelques semaines dans un camp de concentration]. Le 29 mars 1943 le ReichsSicherheitsHauptAmt (RSHA) - Office central de sécurité du Reich - a donné l'ordre de déporter les Tsiganes du Nord de la France et de Belgique.

Les premières arrestations connues ont lieu à Tournai le 22 octobre 1943 et s'échelonnent jusqu'en décembre. Les familles sont transférées au camp de rassemblement des juifs à Malines. 352 Tsiganes de diverses nationalités sont déportés par le convoi Z parti le 15 janvier 1944. C'est le seul convoi de déportation collective à destination d'AuschwitzBirkenau à partir de cette Zone. Ils étaient vanniers, maquignons, étameurs, musiciens, forains ou artistes de cirque (document suivant). La plus jeune n'avait que 38 jours et le plus âgé avait 85 ans. 266 femmes et enfants de moins de 15 ans ont constitué plus des trois quarts de ce transport. 


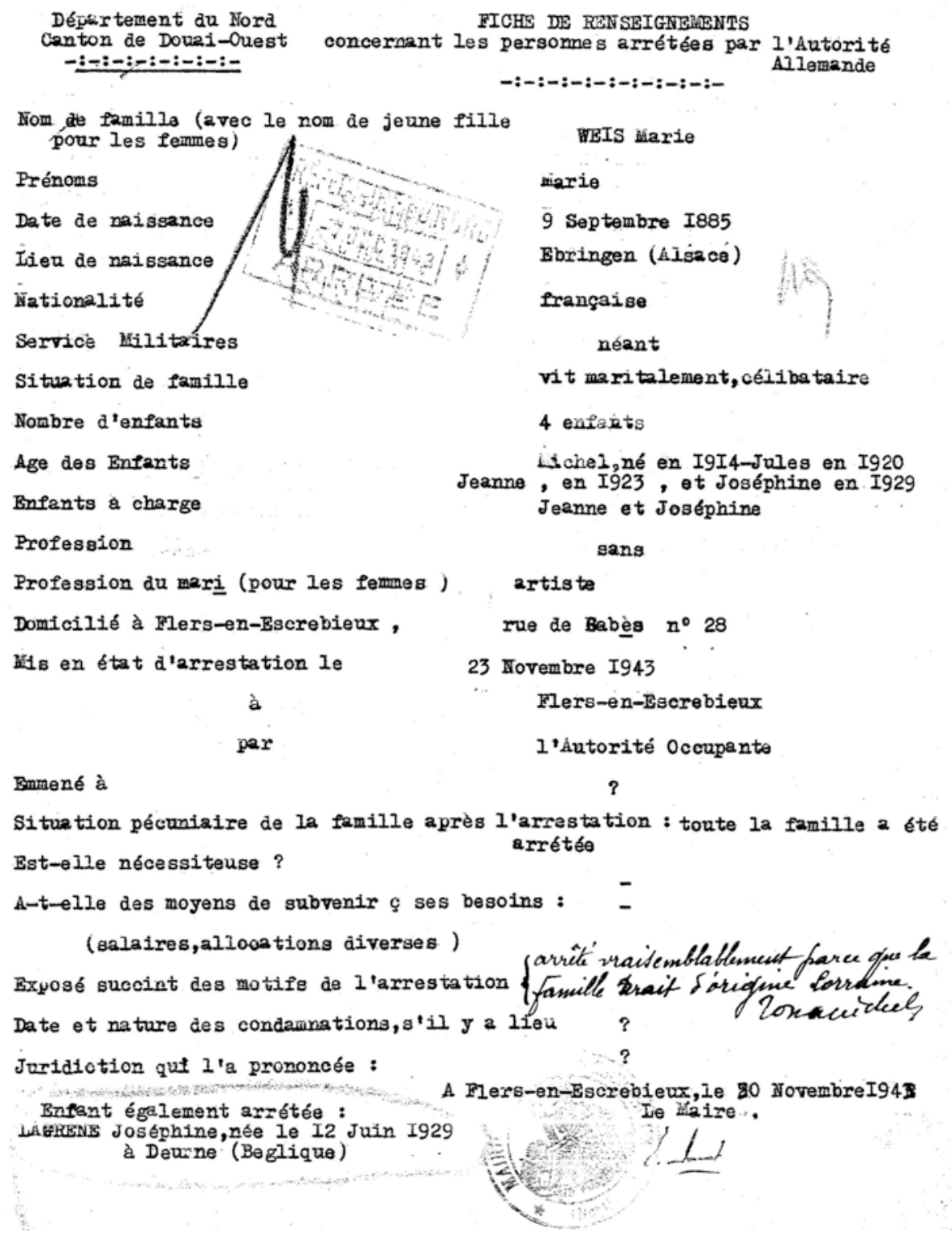

Arrestation de Marie Weiss

AD59, 1W1880

À leur entrée à Birkenau, les Tsiganes ne sont pas triés à la différence des juifs qui sont sélectionnés dès leur arrivée dans ce centre de la mise à mort industrielle. Ils sont immatriculés, tatoués et internés dans le camp des familles qui se révèle très vite être un véritable mouroir. 


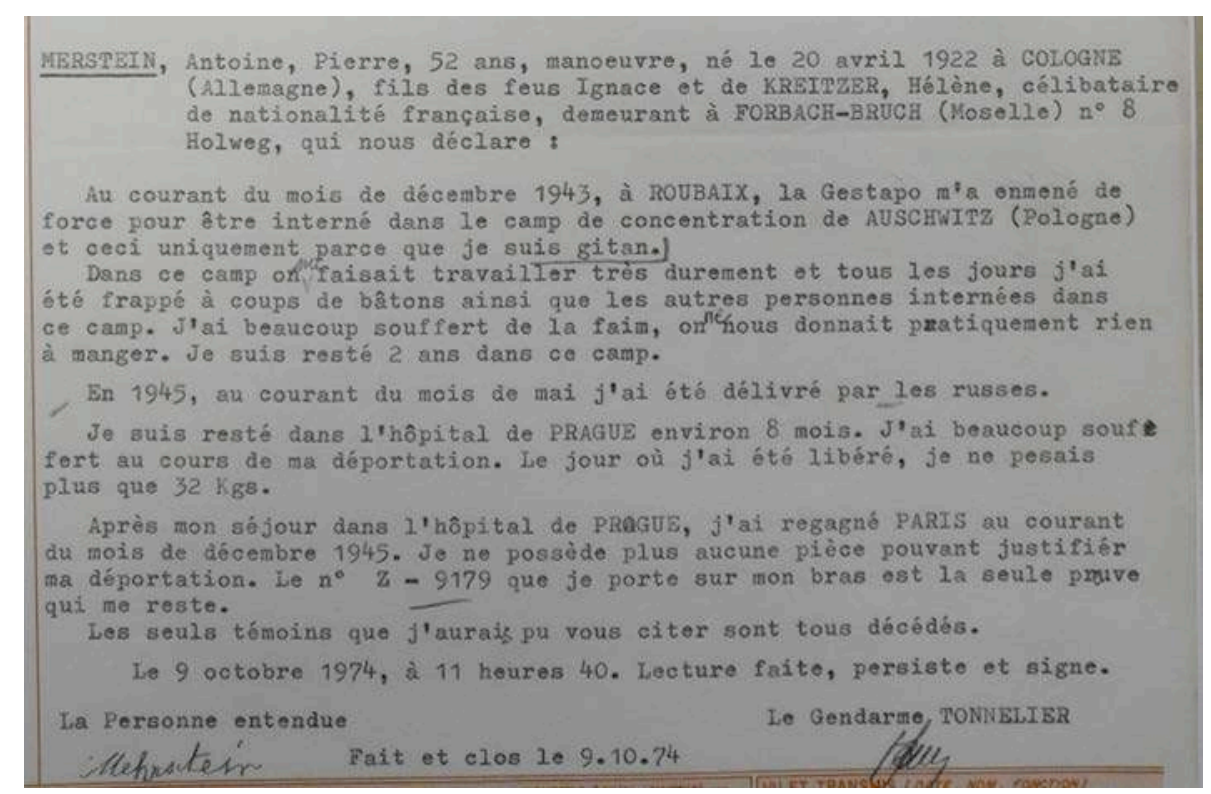

Témoignage d'Antoine Mehrstein BAVCC Caen, 77950

Le pic des décès atteint son point culminant en mars 1944. Plus de la moitié du convoi a succombé entre le 17 janvier et le 31 juillet 1944 . Les Tsiganes de la Zone rattachée arrivent en fait à Birkenau à une période où leur extermination est déjà programmée. C'est ainsi que les 3000 Tsiganes restés dans le camp parce que jugés inaptes au travail, sont éliminés dans la nuit du 2 au 3 août 1944.

Les 68 Tsiganes du convoi $\mathrm{Z}$ qui avaient dépassé le cap de survie des trois mois, ont été transférés entre temps dans les camps du Reich et leurs Kommandos au service de l'économie de guerre: Buchenwald, Dora, Ellrich, Ohrdruf... pour les hommes, Ravensbrück, Schlieben, Taucha, Altenburg... pour les femmes. Seuls 19 hommes et 13 femmes du convoi $\mathrm{Z}$ de Malines sur 352 ont survécu, soit moins de $10 \%$.

$* * * * * * * * * * * * * * * * *$

Les départements du Nord et du Pas-de-Calais ont vécu une guerre différente du reste de la France. Ses spécificités trouvent leur origine dans leur histoire concomitante à leur ancienne appartenance à l'Artois et à la Flandre, dans leur proximité avec le Royaume-Uni qui fut le plus 
tenace au cours du conflit et là où les Allemands étaient persuadés qu'aurait lieu le débarquement.

L'héritage de la Première Guerre mondiale, l'occupation militaire immédiate et le rattachement à Bruxelles expliquent les capacités de résistance de la population. D'autres facteurs d'une grande diversité sont venus renforcer ces singularités : la densité de la population, la forte structuration de la classe ouvrière avec sa capacité à s'organiser collectivement, son engagement syndical, politique et laïque, une économie puissante, des protestants et des catholiques engagés dans des mouvements visant à réconcilier les ouvriers avec le christianisme sur le terrain. L'ensemble de ces spécificités éclairent les conditions dans lesquelles a eu lieu un sauvetage unique en France, voire en Europe, au nez et à la barbe des Allemands, celui des juifs parqués dans la gare de Lille-Fives en attente de leur déportation. À signaler également que cette région fut la seule à connaître la déportation des Tsiganes en raison de sa proximité avec le Reich allemand.

Tout ce pan de l'histoire septentrionale commence à être plus précisément documenté depuis l'ouverture des archives en 2005, mais également en raison de l'intérêt des historiens - belges et français - et des enseignants pour cette Zone restée longtemps hors champ de la recherche. Les études comparatives sur les chronologies et les modes opératoires entre les différentes Zones (rattachée, Alsace-Lorraine, zone italienne, la Belgique et les Pays-Bas) restent toutefois à étudier en développant une nouvelle approche historiographique. 


\section{Bibliographie}

Boudard Lucien, La Maison des enfants. Château de la Huda, Construire et reconstruire, 2008, Maubeuge, $295 \mathrm{p}$.

Dejonghe Étienne, Le Maner Yves, Le Nord-Pas-de-Calais dans la main allemande. 1940-1944, Lille, Éditions La Voix du Nord, 2019, $2^{\mathrm{e}}$ édition, $431 \mathrm{p}$.

Delmaire Danielle, Actes de la Table Ronde sur : Les "camps de Juifs" ouverts dans le Boulonnais par l'Organisation Todt, Bulletin de MEMOR, $\mathrm{n}^{\circ} 10$, octobre $1989,45 \mathrm{p}$.

Delmaire Danielle, Kleiman Thierry, «Liste des déportés du convoi X », Tsafon, revue d'études juives du Nord, $\mathrm{n}^{\circ}$ 9-10, été-automne 1992, p. 43-66 et «Liste des convois de la déportation partis de Malines, dans lesquels se trouvent des Juifs arrêtés dans le Nord», Tsafon, revue d'études juives $d u$ Nord, $\mathrm{n}^{\circ} 21$, printemps 1995, p. 3-29.

Delmaire Danielle, (en collaboration avec Yves-Marie Hilaire), «Chrétiens et Juifs dans le Nord et Pas-de-Calais pendant la Seconde Guerre mondiale », Revue du Nord, avril-juin 1978, p. 451 à 456.

Delmaire Danielle, «Le cardinal Liénart devant la persécution des Juifs de Lille », dans André Kaspi, Serge Klarsfeld, Georges Wellers (sous la dir.) La France et la Question juive 1940-1944, Paris, S. Messinger, 1981, p. 231 à 248.

Filhol Emmanuel, Hubert Marie-Christine, Les Tsiganes en France. Un sort à part 1939-1946, Paris, Perrin, 2009, $420 \mathrm{p}$.

Heddebaut Monique, Des Tsiganes vers Auschwitz - Le convoi $Z$ du 15 janvier 1944, coll. Ces oubliés de l'Histoire, Paris, éd. Michel Reynaud-Tirésias, 336 p. Site : www. editionstiresias.com

Heddebaut Monique, «Sans armes face à la rafle du 11 septembre 1942 », Tsafon, revue d'études juives du Nord, n ${ }^{\circ} 70$ automne 2015 hiver 2016, p. 119-168.

Heddebaut Monique, «Persécutions raciales dans le Douaisis pendant la Seconde Guerre mondiale. Juifs et Tsiganes », Tsafon, revue d'études juives du Nord, hors-série $\mathrm{n}^{\circ}$ 4, octobre 2008, 143 p.

Hilaire Yves-Marie, «Les protestants du Nord et la Seconde Guerre Mondiale », Revue du Nord, avril-juin 1978, p. 445-450.

Lafitte Michel, «L'UGIF fut-elle un obstacle au sauvetage ? », Jacques Semelin éd., La résistance aux génocides. De la pluralité des actes de sauvetage. Paris, Presses de Sciences Po, 2008, p. 411-424. 
Mandelbaum Daniel, Mandelbaum-Bleibtreu Jacqueline, «Le 11 septembre 1942. Les cheminots et les déportés juifs en gare de FivesLille », Tsafon, revue d'études juives $d u$ Nord, $\mathrm{n}^{\circ} 76$, aut 2018-hiver 2019, p. 169-176. Il s'agit du témoignage des enfants d'un couple sauvé par un cheminot.

Mariot Nicolas, Zalc Claire, Face à la persécution : 991 Juifs dans la guerre, Paris, éd. Odile Jacob, 2010, 302 p.

Pasche Marcel, Friedrich Günther - Une figure lumineuse, dans Liens protestants, Protestants résistants, Cahier ${ }^{\circ} 1$, juin 2014, p. 2-3.

Schram Laurence, Dossin - L'antichambre d'Auschwitz, Bruxelles, Éd. Racine, 2017, 348 p.

Semelin Jacques, Persécutions et entraides dans la France occupée, Paris, Les Arènes-Seuil, 2013, 903 p.

Sueur Marc, «Des chrétiens dans la Résistance : l'exemple de la Voix du Nord », Revue du Nord, tome 60, $\mathrm{n}^{\circ}$ 238, juillet-septembre 1978, p. 629-638.

Thiery Laurent, La répression allemande dans le Nord de la France (1940-1944), Villeneuve d'Ascq, Presses Universitaires du Septentrion, 2013, $366 \mathrm{p}$.

Engagés volontaires juifs étrangers dans les armées françaises durant les deux guerres mondiales, catalogue publié à l'occasion de l'exposition organisée par Le Mémorial de la Shoah de novembre 2014 à mars 2015.

Memorial book. The Gypsies at Auschwitz-Birkenau, Musée d'État d'Auschwitz-Birkenau \& K. G. Saur Munich, 1993, 2 vol., 1672 p.

Les Voix du Nord clandestines, Éditions La Voix du Nord, 2011.

$* * * * * * * * * * * * * * * *$

Liste des abréviations

AD59 : Archives départementales du Nord

AML : Archives municipales de Lille

BAVCC : Bureau des Archives des Victimes des Conflits Contemporains 